\title{
PALUDISME POST-TRANSFUSIONNEL
}

\author{
Données statistiques partielles. \\ Approche prophylactique par le dépistage \\ des donneurs de sang à risque
}

\author{
J.-P. SALEUN*, P. DEROFF, A.-M. SIMITZIS**, \\ S. FADE*, Cl. FEREC*
}

RÉSUMÉ. Les auteurs démontrent la réalité du paludisme post-transfusionnel, définissent les espèces plasmodiales en cause et insistent sur la responsabilité de $\boldsymbol{P}$. falciparum, agent de complications gravissimes.

Une approche prophylactique est proposée par la recherche des anticorps antipalustres chez les donneurs de sang à risque. La technique utilisée fait appel à l'I. F. I. en recourant à des réactifs commercialisés par l’Institut Bio-Mérieux sous le nom de Falciparum Spot I. F. Le protocole du dépistage laisse une grande place à l'interrogatoire mais les résultats obtenus sont satisfaisants et semblent améliorables par l'utilisation d'anticorps monoclonaux spécifiques.

Mots-clés : Paludisme. Transfusion. P. falciparum. Donneurs de sang à risque. Zones d'endémie.

\section{Post-transfusion malaria: partial statistical data; prophylactic approach by the screening of donors at risk.}

SUMMARY. The authors demonstrate the reality of post-transfusion malaria, define the plasmodial species involved, and insist on the responsability of Plasmodium falciparum in the occurrence of major complications.

A prophylactic approach is proposed, by researching anti-malarial antibodies in donors at risk. An I. F. A. (Indirect Immunofluorescence Assay) is performed (Falciparum Spot I. F.TM, BioMérieux, Lyon, France).

The screening procedure relies heavily on medical history taking, but subsequent results are satisfying; moreover it seems they could be bettered by using specific monoclonal antibodies.

Key-words: Malaria. Transfusion. P. falciparum. Donors at risk. Endemic areas.

Deux enquêtes successives, pratiquées en 1975 et 1980, nous ont démontré que le paludisme post-transfusionnel était moins rare en France que nous ne l'admettions.

* Centre Départemental de Transfusion Sanguine, B. P. 454, F 29275 Brest.

** Service de Bactério-Viro-Parasitologie, C. H. U., F 29200 Brest.

Accepté le 16 juin 1986. 
L'utilisation des questionnaires adressés aux Établissements de Transfusion Sanguine et l'exploitation de la littérature médicale nous ont permis de recenser 110 cas de paludisme (tableau I) transmis par transfusion entre 1960 et 1979 (Saleun, 1981).

Tableau I. - Cas de Paludisme transmis par transfusion entre 1960 et 1979.

\begin{tabular}{cccccc}
\hline $\begin{array}{c}\text { Cas } \\
\begin{array}{c}\text { ne mentionnant } \\
\text { pas l'année }\end{array}\end{array}$ & $\begin{array}{c}1960- \\
1964\end{array}$ & $\begin{array}{l}1965- \\
1969\end{array}$ & $\begin{array}{l}1970- \\
1974\end{array}$ & $\begin{array}{l}1975- \\
1979\end{array}$ & Total \\
\hline 7 & 13 & 11 & 29 & 50 & 110 \\
\hline
\end{tabular}

Ces constatations nous incitaient à conclure que le paludisme transfusionnel était en nette recrudescence (24 cas observés entre 1960 et 1969 et 79 cas entre 1970 et 1979$)$.

Nous savions, de surcroît, que les chiffres rapportés étaient nettement inférieurs à la réalité puisque :

- d'une part, tous les organismes transfusionnels interrogés n'avaient pas répondu à notre questionnaire,

- d'autre part, ces organismes ne possédant pas le contrôle de la transfusion, certains accidents ne leur étaient pas rapportés.

Nous attribuions cette recrudescence des complications post-transfusionnelles à plusieurs causes :

- l'augmentation des émigrants,

- la multiplication des voyages touristiques ou d'affaires en zone d'endémie palustre,

- le développement de la thérapeutique transfusionnelle avec l'utilisation de plus en plus grande de concentrés érythrocytaires et de fractions labiles pouvant contenir des globules rouges.

\section{Espèces plasmodiales responsables}

Trois espèces d'hématozoaires semblent préférentiellement impliquées : Vivax, Malariae et Falciparum et, à partir de 103 observations parfaitement documentées, nous pouvions établir le tableau II (Saleun, 1981).

La responsabilité de $P$. falciparum se confirmait donc au fil des ans; passant de $15,4 \%$ durant la période 1960-1964 à 45,5\% de 1965 à 1969 , puis à $79 \%$ de 1970 à 1974, pour atteindre $84 \%$ de 1974 à 1979 .

En trois décennies, nous assistions donc à un changement de prépondérance des espèces plasmodiales en cause puisque Bruce-Chwatt (1974), entre 1950 et 1972, 
TABleau II. - Espèces plasmodiales responsables.

\begin{tabular}{|c|c|c|c|c|c|}
\hline Espèces & $\begin{array}{l}1960- \\
1964\end{array}$ & $\begin{array}{l}1965- \\
1969\end{array}$ & $\begin{array}{l}1970- \\
1974\end{array}$ & $\begin{array}{l}1975- \\
1979\end{array}$ & Total \\
\hline $\begin{array}{l}\text { Vivax } \\
\text { Malariae } \\
\text { Falciparum } \\
\text { Non typé }\end{array}$ & $\begin{array}{l}6(45,1 \%) \\
2(15,4 \%) \\
2(15,4 \%) \\
3(23,0 \%)\end{array}$ & $\begin{array}{ll}0 & 0 \\
5 & (45,5 \%) \\
5 & (45,5 \%) \\
1 & (9,0 \%)\end{array}$ & $\begin{array}{rl}2 & (7,0 \%) \\
4 & (13,9 \%) \\
23 & (70,0 \%) \\
0 & 0\end{array}$ & $\begin{array}{rr}2 & (4,0 \%) \\
4 & (8,0 \%) \\
42 & (84,0 \%) \\
2 & (4,0 \%)\end{array}$ & $\begin{array}{r}10(9,8 \%) \\
15(14,7 \%) \\
72(69,6 \%) \\
6 \quad(5,9 \%)\end{array}$ \\
\hline
\end{tabular}

colligeait 1246 cas de paludisme transfusionnel et $P$. malariae était impliqué dans $67 \%$ des cas.

Plasmodium ovale n'est mentionné dans aucune observation. Ceci est peut-être dû au fait que, dans de nombreux cas, l'infection par $P$. ovale peut être masquée par une autre espèce plasmodiale à développement plus rapide (l'association $P$. ovale- $P$. falciparum est particulièrement fréquente en Afrique intertropicale) (Ambroise-Thomas).

Ces deux notions : recrudescence des cas de paludisme post-transfusionnel et prédominance de l'espèce Falciparum pouvant être responsable de complications gravissimes puisque impliquée dans les accès pernicieux, nous ont amenés à pratiquer, dès 1981, le dépistage des donneurs de sang susceptibles de transmettre $P$. falciparum par la recherche, chez ces donneurs, d'Anticorps antipalustres. Cette démarche nous paraissait judicieuse car, selon A. Capron, le paludisme humain offre, en effet, la caractéristique d'une immunité non stérilisante de type I, c'est-à-dire que la réponse immune est le plus souvent liée à la persistance du parasitisme donc de l'hématozoaire à un seuil contrôlé (Bach, 1986).

A cet effet, nous avons tout d'abord collaboré avec les Laboratoires du Département de Parasitologie des Maladies Tropicales du C. H. U. de La Pitié-Salpêtrière (Druilhe, 1978) et utilisé une technique d'électrosynérèse. Mais, dans le même temps, une nouvelle présentation de l'antigène plasmodial nous a permis de mettre en œuvre une technique d'I. F. I. déjà préconisée, depuis 1976, par AmbroiseThomas (1976).

\section{Réactifs et technique (Deroff, 1982)}

Les réactifs sont commercialisés par l'Institut Bio-Mérieux sous le nom de Falciparum Spot I. F. L'antigène est présenté sous forme de dépôts d'hématies parasitées par des $P$. falciparum obtenus à partir de culture in vitro. Les étalements sont séparés en 10 cases pouvant recevoir autant de dilutions de sérums à tester. Ils sont prêts à l'emploi et se conservent à $4^{\circ} \mathrm{C}$.

La case $n^{0} 1$ est recouverte de tampon P. B. S., sans recevoir de sérum; elle servira de témoin négatif. La case adjacente $n^{0} 2$ reçoit $10 \mu l$ d'un témoin positif, 
dilué au $1 / 20^{\mathrm{e}}$ en P. B. S., provenant de la sérothèque du Centre. Les autres cases sont recouvertes de $10 \mu l$ de chaque échantillon de sérum à tester dilué au $1 / 20^{\circ}$.

Les préparations sont alors placées 30 minutes à $37^{\circ} \mathrm{C}$ en chambre humide puis, cette incubation terminée, lavées deux fois en P. B. S. et séchées par une aspiration à la trompe à vide le long de la circonférence de chaque case, suivie d'un soufflage d'air chaud. Chaque case est ensuite recouverte de $25 \mu l$ de conjugué convenablement dilué contenant du Bleu Evans au 1/10000e, et les lames remises en incubation 30 minutes à $37^{\circ} \mathrm{C}$. Après un dernier lavage en P. B. S., puis en eau distillée, les lamelles sont montées et la lecture est faite.

Comme le préconisait l'Institut Bio-Mérieux, nous avons adopté comme limite inférieure de positivité la dilution du $1 / 20^{\mathrm{e}}$, d'ailleurs communément admise par les auteurs manipulant un antigène homologue. Le microscope utilisé est un microscope en épifluorescence.

\section{Méthodes (Deroff, 1982)}

Sont considérés comme donneurs à risque tous les sujets ayant séjourné en zone d'endémie palustre depuis moins de trois ans. Les donneurs sont sélectionnés lors de l'interrogatoire médical qui précède le don par une simple question : "Avez-vous quitté l'Europe depuis moins de trois ans ? ". Environ $10 \%$ des donneurs répondent par l'affirmative. Nous nous enquérons alors de leur destination en nous rapportant aux cartes d'évaluation du paludisme constamment remises à jour par l'Organisation Mondiale de la Santé. Si le pays dans lequel ils se sont rendus appartient à une zone d'endémicité, leur prélèvement fait l'objet d'un séro-diagnostic. Nous ne tenons aucun compte, ni d'une possible symptomatologie palustre antérieure, ni d'une éventuelle chimioprophylaxie.

Les produits sanguins trouvés positifs à la dilution seuil du $1 / 20^{\mathrm{e}}$ sont alors éliminés du circuit transfusionnel direct. Par contre, une sérologie négative exclut la notion d'un paludisme évolutif. Toutefois, si le donneur n'a quitté les zones d'endémie que depuis peu de temps, il faut tenir compte d'une période de latence pré-sérologique absolument silencieuse durant laquelle Ia négativité de l'examen par I. F. n'offre absolument aucune garantie. Cette phase de latence est évaluée à quatre mois, si bien que tous les prélèvements provenant de sujets rentrés depuis moins de quatre mois d'un pays impaludé, sont également éliminés du circuit transfusionnel direct, même si le séro-diagnostic est négatif. Seul leur plasma est utilisé pour le fonctionnement.

\section{Résultats}

\section{FrÉQuences observées}

Sur une période de quatre années (juillet 1981 à juillet 1985), nous avons prélevé 158788 unités de sang. 
Après interrogatoire, 1747 sujets ont été considérés comme donneurs à risque, ce qui représente $1,10 \%$ du total des prélèvements : 107 dépistages en I. F. se sont avérés positifs, soit $6,12 \%$ des dons à risque et seulement $0,67 \%$ unités de sang prélevées.

\section{Observations}

Dans notre statistique, nous n'avons trouvé aucune différence significative des fréquences de sujets séro-positifs, selon que leur retour en Europe soit inférieur à quatre mois, ou compris entre quatre mois et deux ans (tableau III).

Tableau III. - Fréquence de sujets séro-positifs selon le délai de retour en Europe.

\begin{tabular}{cccc}
\hline & $\begin{array}{c}\text { I. F. A. } \\
\text { négatives }\end{array}$ & $\begin{array}{c}\text { I. F. I. } \\
\text { positives }\end{array}$ & $(\%)$ \\
\hline $\begin{array}{c}\text { Retour en Europe depuis } \\
4 \text { mois au moins }\end{array}$ & 387 & 33 & $(8,53 \%)$ \\
$\begin{array}{c}\text { Retour depuis plus de } \\
4 \text { mois et moins de } \\
3 \text { ans } \\
\begin{array}{c}\text { Date de retour imprécise } \\
\text { dans nos archives }\end{array}\end{array}$ & 751 & 48 & $(6,39 \%)$ \\
& 502 & 26 & $(5,18 \%)$ \\
\hline
\end{tabular}

Mais cette constatation n'apporte aucun renseignement sur la durée de la phase pré-sérologique puisque nous ne connaissons pas la durée exacte du séjour en zone impaludée.

Il semble toutefois que la durée d'éviction de quatre mois après le retour en France soit suffisante pour éviter de transmettre un paludisme transfusionnel à $P$. falciparum. Dans notre étude néanmoins, chez deux donneurs, les séro-conversions n'ont été décelées que cinq et dix-huit mois après leur retour en métropole, alors que des recherches d'anticorps, effectuées antérieurement lors de dons consentis par les intéressés dès leur arrivée en Bretagne et plusieurs mois après, s'étaient avérées négatives. Ces séro-positivités à retardement nous incitent à contrôler les sujets à risque, à chacun de leur don, durant un délai de trois ans. L'utilisation d'anticorps monoclonaux immuns spécifiques, pour la mise en évidence chez ces donneurs d'une éventuelle parasitémie, serait à notre avis très bénéfique car elle permettrait, en cas de négativité, de les réintégrer dans le circuit transfusionnel classique (Wells, 1985).

Comme L. Wells et Ala, nous pensons que la séro-positivité peut persister très longtemps après l'impaludation potentielle et la notion de "cicatrice sérologique palustre " peut être évoquée. Trois sujets trouvés séro-positifs en 1981 ont accepté d'être contrôlés plusieurs fois par an, sans être donneurs. Tous les trois sont demeu- 
rés positifs et, afin d'éviter des prélèvements intempestifs, nous les avons orientés vers d'autres techniques de prélèvement, telle que la plasmaphérèse destinée au fractionnement.

Il faut aussi considérer que la longue persistance des anticorps antipalustres peut être le fait d'une communauté antigénique entre les divers types de Plasmodium et le dépistage que nous effectuons n'est peut-être pas spécifique du seul Falciparum. Les positivités que nous découvrons peuvent relever d'infestations à $P$. vivax ou malariae.

\section{Origine géographique des donneurs responsables (tableau IV)}

Nous nous sommes efforcés de regrouper les divers pays en grands ensembles régionaux, individualisés à l'intérieur de chaque continent par des caractéristiques géographiques et climatiques (Gentilini).

Certaines régions, en particulier l'Afrique Intertropicale, demeurent à très haut risque, les régions du Sahel paraissant même plus dangereuses que les régions forestières. Cette prépondérance de l'Afrique tient aux échanges privilégiés entretenus avec la France.

L'Asie du Sud-Est, impliquée fondamentalement dans les statistiques de l'Organisation Mondiale de la Santé par le nombre de cas de paludisme déclarés, ne possède pas la même importance dans nos résultats, sans doute en raison de l'éloignement. L'émigration en provenance de ces pays est limitée et les voyages touristiques peu développés.

Depuis dix-huit mois environ, nous avons inclu l'Afrique du Nord dans notre liste de zones à risque. Nous savions que le paludisme sévissait dans certaines oasis du Sud Algérien mais le Maroc nous paraissait épargné. Sept sérologies positives chez des sujets en provenance de ce pays nous ont rendus prudents (il serait, bien entendu, nécessaire de vérifier leur destination réelle et d'être assurés qu'ils n'ont pas transité par d'autres pays.

\section{Conclusion}

Il faut insister sur les limites d'un protocole basé, au départ, sur un simple interrogatoire; les renseignements que nous recueillons sont quelquefois erronés et le personnel assez fluctuant de nos équipes peut omettre de poser certaines questions ou de préciser quelques détails.

Nous savons aussi qu'une telle procédure ne permet pas d'éliminer les rares cas de paludisme d'importation, mais ceux-ci sont véritablement peu nombreux. En 1983, en Europe, quatre cas de paludisme seulement ont été imputés à des vecteurs en provenance de zones impaludées (Bruaire, 1985).

Nous devons enfin reconnaître que la technique d'I. F. I. que nous utilisons 
Tableau IV. - Origine géographique des donneurs responsables.

\begin{tabular}{|c|c|c|c|c|c|c|}
\hline & \multicolumn{2}{|c|}{$\begin{array}{l}\text { Nb. donneurs } \\
\text { testés }\end{array}$} & \multicolumn{4}{|c|}{ I. F. I. positives } \\
\hline & Pays & Régions & & Pays & & égions \\
\hline $\begin{array}{l}\text { ORIGINE INDÉTERMINÉE } \\
\text { (séjours dans plusieurs } \\
\text { pays d'endémie) }\end{array}$ & & 145 & & & 9 & $6,21 \%$ \\
\hline $\begin{array}{l}\text { AFRIQUE INTERTROPICALE } \\
\text { MADAGASCAR } \\
\text {. Mauritanie, Sénégal, } \\
\text { Gambie, Guinée, Libéria } \\
\text {. Burkina Fasso, Mali, } \\
\text { Niger, Tchad } \\
\text { Golfe du Bénin } \\
\text {. Zone Équatoriale } \\
\text {. Afrique de l'Est } \\
\text {. Madagascar }\end{array}$ & $\begin{array}{r}186 \\
42 \\
111 \\
113 \\
27 \\
18\end{array}$ & 497 & $\begin{array}{r}13 \\
5 \\
11 \\
8 \\
2 \\
3\end{array}$ & $\begin{array}{r}6,99 \% \\
11,90 \% \\
9,91 \% \\
7,08 \% \\
7,41 \% \\
16,67 \%\end{array}$ & 42 & $8,45 \%$ \\
\hline 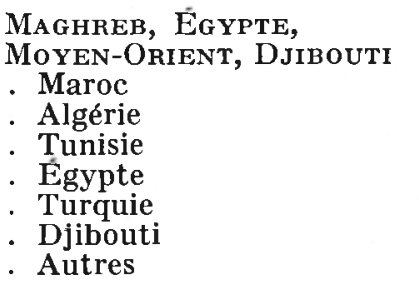 & $\begin{array}{r}36 \\
7 \\
8 \\
26 \\
11 \\
125 \\
39\end{array}$ & 252 & $\begin{array}{l}7 \\
2 \\
0 \\
0 \\
1 \\
5 \\
4\end{array}$ & $\begin{array}{r}19,44 \% \\
28,57 \% \\
\\
9,09 \% \\
4,00 \% \\
10,25 \%\end{array}$ & 19 & $7,53 \%$ \\
\hline $\begin{array}{l}\text { Caraïbes, Amérique } \\
\text { cEntrale } \\
\text { Haïti } \\
\text {. Antilles } \\
\text {. Amique } \\
\text { Amérique Centrale }\end{array}$ & $\begin{array}{l}25 \\
17 \\
40 \\
19\end{array}$ & 101 & $\begin{array}{l}2 \\
1 \\
1 \\
2\end{array}$ & $\begin{array}{r}8,00 \% \\
5,88 \% \\
2,50 \% \\
10,53 \%\end{array}$ & 6 & $5,94 \%$ \\
\hline $\begin{array}{l}\text { Amérique dU SUd } \\
\text {. Brésil } \\
\text {. Guyane } \\
\text {. Autres }\end{array}$ & $\begin{array}{l}25 \\
26 \\
35\end{array}$ & 86 & $\begin{array}{l}1 \\
3 \\
0\end{array}$ & $\begin{array}{r}4,00 \% \\
11,54 \%\end{array}$ & 4 & $4,65 \%$ \\
\hline $\begin{array}{l}\text { SOUS-CONTINENT INDIEN, } \\
\text { EXTRÉME-ORIENT, } \\
\text { OcÉANIE Sri Lanka } \\
\text { Indes, Sri Lanka } \\
\text {. Indonésie } \\
\text { Océan Indien } \\
\text {. Autres }\end{array}$ & $\begin{array}{l}50 \\
85 \\
33 \\
37\end{array}$ & $\overline{1286}$ & $\begin{array}{l}1 \\
3 \\
1 \\
0\end{array}$ & $\begin{array}{l}2,00 \% \\
3,53 \% \\
3,03 \%\end{array}$ & $\overline{85}$ & $2,44 \%$ \\
\hline
\end{tabular}


présente l'inconvénient de ne détecter que la conséquence d'une impaludation, et la mise en évidence des anticorps antipalustres chez un donneur ne traduit que d'une manière toute relative un éventuel pouvoir contaminant.

Cependant, nous pensons que notre protocole, peu onéreux et relativement simple, offre de bonnes garanties de sécurité pour le dépistage des donneurs à risque. En l'associant, chez les donneurs trouvés séro-positifs, à la recherche des antigènes particulaires de $P$. falciparum par I. F. I. à l'aide d'anticorps monoclonaux (Prouo, 1986; Soulier, 1982), nous obtiendrions des résultats excellents permettant la récupération de nombreux culots globulaires éliminés abusivement par notre méthode.

\section{BIBLIOGRAPHIE}

Ambroise-Thomas P., Carnevale P., Felix H., Mouchet J. : Encyclopédie Médico-Chirurgicale, Paris, 8089 A. 10.

Ambroise-Thomas P. : Prévention du Paludisme post-transfusionnel par séro-dépistage des porteurs latents de plasmodium parmi les donneurs de sang. Rev. Fr. Transfus. Immunohematol., $1976,19,331$.

BAсH J. F. : Immunologie a L’immunité anti-parasitaire ». Flammarion Médecine-Sciences, Paris, 1986, $3^{\mathrm{e}}$ éd., Chap. 23, p. 641 .

Bruaire M., Jestin C., Perrin J., Chauvin M. A. : Bulletin épidémiologique hebdomadaire. Direction Générale de la Santé, 1985, nº 46.

Bruce-Chwatt L. J. : Transfusion Malaria. Bull. Wald. Heth. Org., 1974, 3, 337.

Deroff P., Reguer M., Simitzis A. M., Boudon A., Saleun J. P. : Dépistage des donneurs de sang susceptibles de transmettre P. falciparum. Rev. Fr. Tranfus. Immunohematol., 1982, $25,3$.

Druilhe P., Monjour L., Richard-Lenoble D., Gentilini M. : L'électro-immunodiffusion sur membrane d'acétate de cellulose pour le diagnostic sérologique du paludisme humain. Pathol. Biol., 1978, 26, 169 .

Gentilini M., Brousse G., Amat-Roze J. M. : Risques infectieux et parasitaires. Documentation Roche.

Prou O.: Mise en évidence de Plasmodium falciparum par immuno-fluorescence indirecte à l'aide d'anticorps monoclonaux murins spécifiques. Rev. Fr. Transfus. Immunohematol., 1986, $28,6$.

Saleun J. P., Colin F., Deroff P., Vicariot M. : Paludisme transfusionnel en France de 1960 à 1979. Nouv. Pres. Med., 1981, 12, 981.

Soulier J. P., Lee H., Prou O., Raymondjean N., Dubarry M., Druilhe P. : Anticorps monoclonaux anti-Plasmodium falciparum. Rev. Fr. Transfus. Immunohematol., 1982, 25, 4.

Wells L., Ala F. A. : Malaria and Blood Transfusion. Lancet, 1985, I, 1317. 